\title{
Reciprocity relation between photovoltaic quantum efficiency and electroluminescent emission of solar cells
}

\author{
Uwe Rau* \\ IEF-5, Photovoltaics, Forschungszentrum Jülich, 52425 Jülich, Germany \\ (Received 21 September 2006; revised manuscript received 18 June 2007; published 2 August 2007)

\begin{abstract}
A rigorous proof for a reciprocity theorem that relates the spectral and angular dependences of the electroluminescence of solar cells and light emitting diodes to the spectral and angular quantum efficiency of photocarrier collection is given. An additional relation is derived that connects the open circuit voltage of a solar cell and its electroluminescence quantum efficiency.
\end{abstract}

DOI: 10.1103/PhysRevB.76.085303

PACS number(s): 72.10.Bg, 05.60.-k, 84.60.Jt

\section{INTRODUCTION}

Light emitting diodes (LEDs) and solar cells are important optoelectronic devices. Because of their complementary physical action, the transformation of electrical energy into light and transformation of radiation energy into electricity, one would intuitively expect a certain reciprocity between the theoretical laws that determine the physical action of both devices. In fact, a solar cell that has the theoretical maximum power conversion efficiency will also act as an LED with the maximum possible luminescence efficiency. This is because light absorption by the generation of electron-hole pairs is linked via the principle of detailed balance to the complementary action, emission of light by radiative recombination. This restriction of the solar cell concept to the basic physical processes of detailed balance between light absorption and radiative recombination makes the elegance of the Shockley-Queisser (SQ) approach ${ }^{1}$ for the calculation of the limiting efficiency of photovoltaic power conversion.

Although a perfect solar cell would also be a perfect LED, in reality neither device exists. However, excellent devices that come relatively close to the respective ideal limits are to be found. It is interesting to note that high-efficiency silicon solar cells, i.e., those which approach the SQ radiative limit, can be used as LEDs with essentially the same design. ${ }^{2}$ These highly sophisticated devices achieve an external (LED) quantum efficiency $\mathrm{EQE}_{L E D} \approx 1 \%$, where $\mathrm{EQE}_{L E D}$ is defined as the ratio between the number of photons emitted from the surface of the sample and the number of recombining electron-hole pairs. In contrast, the photovoltaic $\mathrm{EQE}_{P V}\left(E_{\gamma}\right)$ of a solar cell is a spectral quantity defined as the ratio of collected charge carriers to the number of photons with energy $E_{\gamma}$ incident on the cell's surface. $\mathrm{EQE}_{L E D} \approx 1 \%$ is a highly respectable value for silicon devices but small when compared to LEDs made from organic semiconductors ${ }^{3}$ with $\mathrm{EQE}_{L E D} \approx 15 \%{ }^{4}$ In contrast, solar cells made from organic materials ${ }^{5}$ have power conversion efficiencies hardly exceeding $2 \%$ (compared to $25 \%$ of the best silicon solar cell ${ }^{6}$. Clearly, at a practical level, below the idealized SQ case, quality requirements for solar cells and LEDs diverge considerably.

The present paper gives a detailed balance theory that embraces also less idealized solar cells and LEDs by adding the aspect of electronic transport to the SQ theory. The cen- tral result is a reciprocity theorem that relates the carrier collection properties of a solar cell to its spectral electroluminescence (EL) emission. This reciprocity approaches the SQ identity of an LED and a solar cell in the limit of infinite charge carrier mobility $\mu_{p}$ and of an infinite nonradiative lifetime $\tau_{n r}$. An additional relation connects the open circuit voltage $V_{\mathrm{OC}}$ of a solar cell and the external quantum efficiency $\mathrm{EQE}_{L E D}$ for the same device operating as an LED.

\section{BASICS}

Let us begin with a rather simple consideration. In thermal equilibrium, each surface element of a solar cell is irradiated from each element of the spherical angle of the ambient with a spectral flux density,

$$
\begin{aligned}
\phi_{e q}\left(E_{\gamma}, \theta\right) & =\phi_{b b}\left(E_{\gamma}\right) \cos (\theta)=\frac{2 E_{\gamma}^{2} /\left(h^{3} c^{2}\right) \cos (\theta)}{\exp \left(E_{\gamma} / k T\right)-1} \\
& \approx \frac{2 E_{\gamma}^{2} \cos (\theta)}{h^{3} c^{2}} \exp \left(\frac{-E_{\gamma}}{k T}\right),
\end{aligned}
$$

corresponding to a blackbody radiation with refractive index $n=1$. In Eq. (1), $h$ is Planck's constant, $c$ the vacuum speed of light, and $k T$ the thermal energy. A portion $a\left(\mathbf{r}_{S}, \theta, \varphi, E_{\gamma}\right)$ depending on the coordinate $\mathbf{r}_{S}$ of the surface element $d S$, the angles of incidence $\theta, \varphi$ (as defined in Fig. 1), and the photon energy $E_{\gamma}$ is absorbed by the solar cell. As a consequence of Kirchhoff's law, this incident radiation is in equilibrium with the radiation emitted from the cell's surface.

Since we are dealing with a solar cell, a fraction of the absorbed photons will generate free electron-hole pairs along the optical path, as illustrated by Fig. 1. These charge carriers will have a probability $F_{C}\left(\mathbf{r}_{S}, \theta, \varphi, E_{\gamma}\right)$ of being collected by the junction of the cell. Therefore, the probability that one photon impinging on the solar cell contributes one elementary charge $q$ to the short circuit current is given by $Q\left(\mathbf{r}_{S}, \theta, \varphi, E_{\gamma}\right)=a F_{C}$, where $a$ is the absorptance of the cell.

Under solar irradiation, an excess flux density $\phi_{\text {sun }}$ impinges on the solar cell, leading to a short circuit current,

$$
J_{S C}=q \int_{\Omega_{\text {sun }}} \int_{E_{\gamma}} \int_{S_{c}} Q\left(\mathbf{r}_{S}, \theta, \varphi, E_{\gamma}\right) \phi_{\text {sun }}\left(\theta, \varphi, E_{\gamma}\right) d \Omega d E_{\gamma} d S,
$$

where the integrals extend over the angle of incidence $\Omega_{\text {sun }}$ and the energy range $\left(E_{\gamma}\right)$ of the solar irradiation and the 


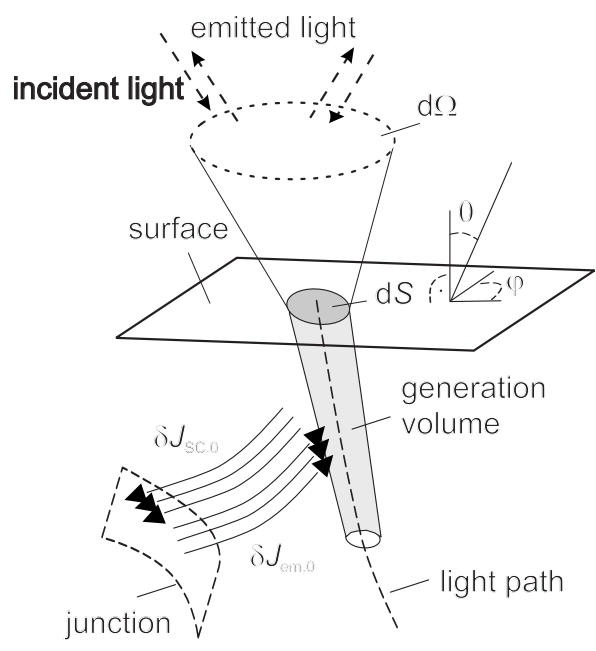

FIG. 1. Illustration of the reciprocal action of light absorption and subsequent carrier collection, complemented by current injection and light emission. In equilibrium, the short circuit current $\delta J_{S C, 0}$ induced by photons entering into the solar cell through the surface element $d S$ within the angle $d \Omega$ is counterbalanced by the saturation current $\delta J_{e m, 0}$. This injection current leads to an emission of photons equilibrating the incident photons.

surface area $S_{c}$ of the cell, respectively. The differential spherical angle $d \Omega$ is defined by $d \Omega=\sin (\theta) d \theta d \varphi$ with the angles $\varphi$ and $\theta$, as shown in Fig. 1, together with the surface element $d S$.

In thermal equilibrium, the solar cell is exposed only to the blackbody radiation from the ambient. Since the device cannot distinguish between photons from the sun and those from the ambient, all equilibrium radiation leads to charge carriers that are collected by the junction. We have therefore to account for an "equilibrium" short circuit current,

$$
J_{S C, 0}=q \int_{\Omega_{c}} \int_{E_{\gamma}} \int_{S_{c}} Q\left(\mathbf{r}_{S}, \theta, \varphi, E_{\gamma}\right) \phi_{e q}\left(E_{\gamma}, \theta\right) d \Omega d E_{\gamma} d S,
$$

resulting from the ambient blackbody flux $\phi_{e q}\left(E_{\gamma}, \theta\right)$ integrated over the full spherical angle $\Omega_{c}=2 \pi$. However, in thermal equilibrium, no net current is flowing through the device. Therefore, we must postulate that the short circuit current $J_{S C, 0}$ is counterbalanced by an equilibrium injection current $J_{e m, 0}=J_{S C, 0}$. Moreover, a similar balance,

$$
\delta J_{e m, 0}=\delta J_{S C, 0}=q Q\left(\mathbf{r}_{S}, \theta, \varphi, E_{\gamma}\right) \phi_{e q}\left(E_{\gamma}, \theta\right),
$$

holds for any component $\delta J_{S C, 0}$ of $J_{S C, 0}$ that is generated from any angular component of the ambient blackbody radiation at any surface element of the cell.

As shown in Fig. 1, we have now introduced a new detailed balance pair, namely, light absorption with subsequent carrier collection complemented by carrier injection with subsequent light emission. The quantity $Q\left(\mathbf{r}_{S}, \theta, \varphi, E_{\gamma}\right)$, if measured under normal incidence and averaged over the cell surface, corresponds to the photovoltaic external quantum efficiency $\mathrm{EQE}_{P V}$ as defined above. Therefore, we denote $Q\left(\mathbf{r}_{S}, \theta, \varphi, E_{\gamma}\right)$ the partial external quantum efficiency. In the
SQ limit, ${ }^{1}$ both quantities, $\mathrm{EQE}_{P V}$ and $Q\left(\mathbf{r}_{S}, \theta, \varphi, E_{\gamma}\right)$, are unity for photons with energies $E_{\gamma} \geqslant E_{g}$ and zero if $E_{\gamma}<E_{g}$.

Next, we postulate that injection of the current component $\delta J_{e m}$ that leads to the radiative emission of photons under an applied voltage $V$ follows an exponential law. Since this dark injection current is superimposed on the component $\delta J_{S C, 0}$, the excess photon flux density $\phi_{\text {em }}$ emitted from the device follows Shockley's diode law, ${ }^{7}$

$$
\phi_{e m}=\frac{\delta J_{e m, 0}}{q} \exp \left(\frac{q V}{k T}\right)-\frac{\delta J_{S C, 0}}{q}=\frac{\delta J_{e m, 0}}{q}\left[\exp \left(\frac{q V}{k T}\right)-1\right]
$$

Note that Secs. III and IV below clarify the conditions for the validity of the postulate leading to Eq. (5). However, the more intuitive approach used at this stage reveals an important property of the detailed balance between photon emission and photon collection in solar cells: the ability of a solar cell to emit photons (expressed by $\delta J_{e m, 0}$ ) is linked directly to optical generation and carrier collection due to the blackbody ambient radiation (expressed by $\delta J_{S C, 0}$ ).

Combining Eqs. (4) and (5) yields

$$
\phi_{e m}\left(\mathbf{r}_{S}, \theta, \varphi, E_{\gamma}\right)=Q\left(\mathbf{r}_{S}, \theta, \varphi, E_{\gamma}\right) \phi_{e q}\left(E_{\gamma}, \theta\right)\left[\exp \left(\frac{q V}{k T}\right)-1\right] \text {, }
$$

and, accordingly, for the integral excess photon flux,

$$
\begin{aligned}
\Phi_{e m}\left(E_{\gamma}\right)= & \int_{\Omega_{C}} \int_{S_{c}} Q\left(\mathbf{r}_{S}, \theta, \varphi, E_{\gamma}\right) \phi_{e q}\left(E_{\gamma}, \theta\right) d \Omega d S \\
& \times\left[\exp \left(\frac{q V}{k T}\right)-1\right] .
\end{aligned}
$$

Thus, Eqs. (6) and (7) allow us to use the partial external quantum efficiency $Q\left(\mathbf{r}_{S}, \theta, \varphi, E_{\gamma}\right)$ of the solar cell to predict the spectral and angular emittance of the device if operated as an LED and vice versa. These equations are, therefore, the differential and the integral version of a reciprocity relation that connects the complementary action of solar cells and LEDs in situations somewhat less ideal than the SQ limit.

\section{RECIPROCITY IN ELECTRONIC TRANSPORT}

In the preceding section, we obtained reciprocity relations (6) and (7) in a rather intuitive way. We now give a rigorous proof, concentrating on the nature of the optoelectronic transport processes compatible with the reciprocity relations. It will be shown that the validity of Eqs. (6) and (7) presupposes an earlier reciprocity theorem derived by Donolato. ${ }^{8}$ This theorem relates the local carrier collection probability $f_{c}$ within the illuminated solar cell to the minority carrier concentration in the same device under forward voltage $V$ in the dark. With equilibrium minority carrier concentrations $p_{0}(\mathbf{r})$ at a site $\mathbf{r}$ in the volume of the absorber material and $p_{0}(j)$ at the collecting junction of the cell, this theorem reads 


$$
\frac{\delta p_{d}(\mathbf{r})}{p_{0}(\mathbf{r})} / \frac{\delta p_{d}(j)}{p_{0}(j)}=f_{c}(\mathbf{r}),
$$

where the quantities $\delta p_{d}(\mathbf{r})$ and $\delta p_{d}(j)$ denote the (dark) excess minority carrier concentrations at coordinate $\mathbf{r}$ and at the junction, respectively. Note that in a $p n$-junction solar cell, $p_{0}(j)$ is the carrier density at the edge of the space charge region. Thus, the junction itself is not part of Donolato's theory, except in defining the boundary conditions. From Shockley's diode law, ${ }^{7}$ we may write $\delta p(j)$ $=p_{0}(j)[\exp (q V / k T)-1]$ and transform Eq. (8) into

$$
\frac{\delta p_{d}(\mathbf{r})}{p_{0}(\mathbf{r})}=f_{c}(\mathbf{r})\left[\exp \left(\frac{q V}{k T}\right)-1\right] \text {. }
$$

A series of generalizations to the original theorem ${ }^{9-12}$ established that Eq. (8) is valid, provided that the minority carrier transport in the solar cell is described by the generalized diffusion equation of del Alamo and Swanson, ${ }^{13}$

$$
-\nabla\left[D(\mathbf{r}) p_{0}(\mathbf{r}) \nabla u(\mathbf{r})\right]+\frac{1}{\tau(\mathbf{r})} p_{0}(\mathbf{r}) u(\mathbf{r})=g(\mathbf{r}),
$$

where $u(\mathbf{r})=\delta p(\mathbf{r}) / p_{0}(\mathbf{r})$ is the normalized excess carrier density and $g(\mathbf{r})$ is the photogeneration rate. In Eq. (10), the diffusion constant $D(\mathbf{r})$ and the minority carrier lifetime $\tau(\mathbf{r})$ may be arbitrary functions of the coordinate $\mathbf{r}$. It is important to note that Eq. (10) is also valid for the diffusion of excitons in the absorber layer of organic solar cells. The validity of the optoelectronic reciprocity for these excitonic-type solar cells (following the definition of $\mathrm{Gregg}^{14}$ ) will be discussed in Sec. VI.

Rau and Brendel ${ }^{15}$ showed that Eq. (8) also holds for a system of discrete sites (e.g., localized states in a semiconductor or dye molecules in a dye-sensitized solar cell) if the occupation dynamics is determined by $g_{i}=\sum_{j} \Theta_{i j} u_{j}$. Here, the symmetric operator $\Theta_{i j}$ accounts for the recombination as well as the transport of charge carriers. The quantity $u_{j}$ denotes the normalized excess carrier density at site $j$, and $g_{i}$ the excess photogeneration rate at site $i$. The symmetry of the operator $\Theta_{i j}$ (as defined in Ref. 15) on the discrete vector space and also the symmetry of the functional operator $\mathbf{L}$ defined by

$$
\mathbf{L} u(\mathbf{r})=-\nabla\left[D(\mathbf{r}) p_{0}(\mathbf{r}) \nabla u(\mathbf{r})\right]+\frac{1}{\tau(\mathbf{r})} p_{0}(\mathbf{r}) u(\mathbf{r}),
$$

which makes up the left hand side of Eq. (10), are the key ingredients to derive Eq. (8). Reference 15 also demonstrates that Donolato's reciprocity theorem is a consequence of the principle of detailed balance.

Up to now, the presence of light plays no role within the prerequisites of this theory except for the photogeneration function $g(\mathbf{r})$. It is therefore unclear whether Eq. (8) is valid in situations where radiative recombination and consequently reabsorption of light (photon recycling) play a non-negligible role in the transport of charge carriers. ${ }^{16,17}$ Therefore, we must analyze more closely the consequences of this type of interaction on the electronic transport.

Let us first recall some basic properties of ray optics by considering two differential area elements $d A_{a}$ and $d A_{b}$ lo-
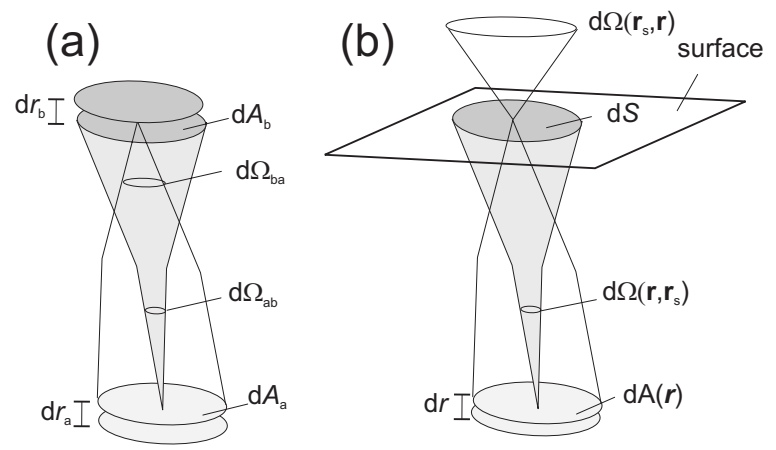

FIG. 2. (a) Illustration of the optical interaction between two volume elements $d A_{a} d \mathbf{r}_{a}$ and $d A_{b} d \mathbf{r}_{b}$. Note that the difference between the spherical angles $d \Omega_{a b}$ and $d \Omega_{b a}$ is due to the assumed difference of the refractive index in $\mathbf{r}_{a}$ and $\mathbf{r}_{b}$. This change of the refractive index is indicated by the kinks in the optical interaction cones. (b) Interaction between a surface element $d S$ and a volume element $d A(\mathbf{r}) d \mathbf{r}$. Light is entering or is emitted within the spherical angle $d \Omega\left(\mathbf{r}_{S}, \mathbf{r}\right)$, whereas $d S$ (located at coordinate $\mathbf{r}_{S}$ ) is seen from the volume element (located at $\mathbf{r})$ within the angle $d \Omega\left(\mathbf{r}, \mathbf{r}_{S}\right)$.

cated at $\mathbf{r}_{a}$ and $\mathbf{r}_{b}$ in the volume of the solar cell, as depicted in Fig. 2(a). Let $d A_{a}=d A_{b}$ and $d \Omega_{a b}, d \Omega_{b a}$ be the spherical angles of $d A_{b}$ seen from $d A_{a}$, and vice versa. Then, the number of photons $\Phi\left(\mathbf{r}_{b}\right)$ per unit area and time arriving at $\mathbf{r}_{b}$ is

$$
\Phi\left(\mathbf{r}_{b}\right)=\phi\left(\mathbf{r}_{b}\right) d \Omega_{b a}=\phi\left(\mathbf{r}_{a}\right) T\left(\mathbf{r}_{a}, \mathbf{r}_{b}\right) d \Omega_{a b},
$$

where $\phi\left(\mathbf{r}_{a}\right), \phi\left(\mathbf{r}_{b}\right)$ are the photon flux densities per unit spherical angle in $\mathbf{r}_{a}, \mathbf{r}_{b}$ and $T\left(\mathbf{r}_{a}, \mathbf{r}_{b}\right)$ is the transmission probability with $0 \leqslant T\left(\mathbf{r}_{a}, \mathbf{r}_{b}\right) \leqslant 1$. Since $T\left(\mathbf{r}_{a}, \mathbf{r}_{b}\right)$ only depends on the optical path between $\mathbf{r}_{a}$ and $\mathbf{r}_{b}$, we have the symmetry relation $T\left(\mathbf{r}_{a}, \mathbf{r}_{b}\right)=T\left(\mathbf{r}_{b}, \mathbf{r}_{a}\right)$. By defining $P\left(\mathbf{r}_{a}, \mathbf{r}_{b}\right)$ $\equiv T\left(\mathbf{r}_{a}, \mathbf{r}_{b}\right) d \Omega_{a b} n\left(\mathbf{r}_{a}\right) / n\left(\mathbf{r}_{b}\right)$, we may rewrite Eq. (12) as

$$
\Phi\left(\mathbf{r}_{b}\right)=\phi\left(\mathbf{r}_{a}\right) P\left(\mathbf{r}_{a}, \mathbf{r}_{b}\right) \frac{n\left(\mathbf{r}_{b}\right)}{n\left(\mathbf{r}_{a}\right)},
$$

where $n\left(\mathbf{r}_{a}\right)$ and $n\left(\mathbf{r}_{b}\right)$ are the refractive indices at $\mathbf{r}_{a}$ and $\mathbf{r}_{b}$. The conservation of étendue allows us to write $n^{2}\left(\mathbf{r}_{a}\right) d \Omega_{a b}$ $=n^{2}\left(\mathbf{r}_{b}\right) d \Omega_{b a}$, and it follows that $P\left(\mathbf{r}_{a}, \mathbf{r}_{b}\right)=P\left(\mathbf{r}_{b}, \mathbf{r}_{a}\right)$.

Let us now assume that the photon flux $\phi\left(\mathbf{r}_{a}\right)$ in $\mathbf{r}_{a}$ is generated by the blackbody radiation of the volume element $d A_{a} d \mathbf{r}_{a}$. The photon flux generated in $\mathbf{r}_{a}$ by unit thickness is then

$$
\frac{d \phi}{d \mathbf{r}_{a}}\left(\mathbf{r}_{a}\right)=\alpha\left(\mathbf{r}_{a}\right) n^{2}\left(\mathbf{r}_{a}\right) \phi_{b b} .
$$

The number of photons arriving at $\mathbf{r}_{b}$ is obtained from Eqs. (13) and (14),

$$
d \Phi\left(\mathbf{r}_{b}\right)=\alpha\left(\mathbf{r}_{a}\right) P\left(\mathbf{r}_{a}, \mathbf{r}_{b}\right) \frac{n\left(\mathbf{r}_{b}\right)}{n\left(\mathbf{r}_{a}\right)} n^{2}\left(\mathbf{r}_{a}\right) \phi_{b b} d \mathbf{r}_{a} .
$$

The equilibrium generation rate $\gamma_{e q}\left(\mathbf{r}_{b}, \mathbf{r}_{a}\right)$ for minority carriers at $\mathbf{r}_{b}$ induced by photons originating from the coordinate $\mathbf{r}_{a}$ is given by the Lambert-Beer law, 


$$
\begin{aligned}
\gamma_{e q}\left(\mathbf{r}_{b}, \mathbf{r}_{a}\right) d \mathbf{r}_{a} & =\alpha\left(\mathbf{r}_{b}\right) d \Phi\left(\mathbf{r}_{b}\right) \\
& =\alpha\left(\mathbf{r}_{b}\right) \alpha\left(\mathbf{r}_{a}\right) n\left(\mathbf{r}_{a}\right) n\left(\mathbf{r}_{b}\right) P\left(\mathbf{r}_{a}, \mathbf{r}_{b}\right) \phi_{b b} d \mathbf{r}_{a} .
\end{aligned}
$$

Note that Eq. (16) represents the detailed (blackbody) balance between $\mathbf{r}_{a}$ and $\mathbf{r}_{b}$. That is, because of the symmetry $\gamma_{e q}\left(\mathbf{r}_{b}, \mathbf{r}_{a}\right)=\gamma_{e q}\left(\mathbf{r}_{a}, \mathbf{r}_{b}\right)$, the blackbody radiation that is generated in $\mathbf{r}_{a}$ and absorbed in $\mathbf{r}_{b}$ equals its detailed balance complement, the blackbody radiation generated in $\mathbf{r}_{b}$ and absorbed in $\mathbf{r}_{a}$.

In order to prove Donolato's theorem in the presence of photon recycling, we now turn to the nonequilibrium situation. Here, the recombination of excess carriers of concentration $\delta p=p-p_{0}$ leads to

$$
\frac{d \Phi_{e x c}\left(E_{\gamma}\right)}{d \mathbf{r}_{a}}\left(\mathbf{r}_{a}\right)=\alpha\left(\mathbf{r}_{a}, E_{\gamma}\right) n^{2}\left(\mathbf{r}_{a}\right) \phi_{b b}\left(E_{\gamma}\right) \frac{\delta p}{p_{0}},
$$

by analogy with Eq. (14). Note that integrating Eq. (17) over energy leads to the definition of the radiative lifetime $\tau_{r}{ }^{18}$

$$
\frac{1}{\tau_{r}}=\frac{4 \pi n^{2} \int \alpha\left(E_{\gamma}\right) \phi_{b b}\left(E_{\gamma}\right) d E_{\gamma}}{p_{0}},
$$

where the factor $4 \pi$ stems from integration over the full spherical angle.

Let us now consider the nonequilibrium photogeneration rate $\gamma\left(\mathbf{r}_{b}, \mathbf{r}_{a}\right)$ at a site $\mathbf{r}_{b}$ that originates from the charge carrier recombination at a site $\mathbf{r}_{a}$ within the solar cell. With the help of Eq. (16) and the definition of the relative excess carrier density $u \equiv \delta p / p_{0}$, we find

$$
\begin{aligned}
\gamma\left(\mathbf{r}_{b}, \mathbf{r}_{a}\right) & =\alpha\left(\mathbf{r}_{b}\right) \alpha\left(\mathbf{r}_{a}\right) n\left(\mathbf{r}_{b}\right) n\left(\mathbf{r}_{a}\right) P\left(\mathbf{r}_{b}, \mathbf{r}_{a}\right) \phi_{b b} u\left(\mathbf{r}_{a}\right) \\
& =\gamma_{e q}\left(\mathbf{r}_{b}, \mathbf{r}_{a}\right) u\left(\mathbf{r}_{a}\right) .
\end{aligned}
$$

Note that the quantity $\gamma\left(\mathbf{r}_{b}, \mathbf{r}_{a}\right)$ still depends on the photon energy. Therefore, we have to integrate $\gamma\left(\mathbf{r}_{b}, \mathbf{r}_{a}\right)$ to obtain $\kappa\left(\mathbf{r}_{b}, \mathbf{r}_{a}\right)=\int \gamma\left(E_{\gamma}, \mathbf{r}_{b}, \mathbf{r}_{a}\right) d E_{\gamma}$, the interaction term between the radiative carrier recombination and subsequent reabsorption of the emitted light. Because of the symmetry $\gamma\left(\mathbf{r}_{b}, \mathbf{r}_{a}\right)$ $=\gamma\left(\mathbf{r}_{a}, \mathbf{r}_{b}\right)$, it follows that

$$
\kappa\left(\mathbf{r}_{b}, \mathbf{r}_{a}\right)=\kappa\left(\mathbf{r}_{a}, \mathbf{r}_{b}\right) .
$$

The symmetry of Eqs. (19) indicates that a relative excess carrier concentration $u\left(\mathbf{r}_{a}\right)$ leads to a generation rate at site $\mathbf{r}_{b}$ that is the same as $u\left(\mathbf{r}_{b}\right)$ would induce at $\mathbf{r}_{a}$.

In the next step, we insert the interaction term $\kappa\left(\mathbf{r}_{b}, \mathbf{r}_{a}\right)$ in Eq. (10) to obtain

$$
\begin{aligned}
& -\nabla\left[D(\mathbf{r}) p_{0}(\mathbf{r}) \nabla u(\mathbf{r})+\frac{1}{\tau(\mathbf{r})} p_{0}(\mathbf{r}) u(\mathbf{r})-\int_{V} \kappa\left(\mathbf{r}, \mathbf{r}^{\prime}\right) u\left(\mathbf{r}^{\prime}\right) d \mathbf{r}^{\prime}\right. \\
& \quad=g(\mathbf{r}),
\end{aligned}
$$

where the integral extends over the cell volume and the lifetime $\tau$ is now the combination of nonradiative and radiative lifetimes with $\tau^{-1}=\tau_{r}^{-1}+\tau_{n r}^{-1}$. Note that the interaction term for photon recycling was already used in the 1970s (Refs. 19 and 20) in the context of the regular diffusion equation for a material with homogeneous electronic and optical properties. The present Eq. (21), however, accounts for a material with arbitrary spatial variations of all relevant electronic and optical quantities.

Because of the symmetry of the integration kernel $\kappa(\mathbf{r}, \mathbf{r} \prime)$ and the symmetry of the operator $\mathbf{L}$ defined in Eq. (11) and entering in Eq. (10), the operator $\mathbf{L}^{\prime}=\mathbf{L}$ $-\int \kappa\left(\mathbf{r}, \mathbf{r}^{\prime}\right) \cdot d V\left(\mathbf{r}^{\prime}\right)$ in Eq. (21) is also symmetric. Note that the symmetry of the operator $\mathbf{L}^{\prime}$ is the only prerequisite for the validity of Eq. (8) (see Ref. 15). Hence, Donolato's reciprocity ${ }^{8}$ holds also in the presence of photon recycling.

At this point, it is interesting to note that we may omit the transport term in Eq. (21) such that we describe a system that is based solely on optical transport such as a photovoltaic fluorescent collector. ${ }^{21}$ We may therefore extend the present theoretical approach to these systems. In fact, it has recently been shown ${ }^{22,23}$ that a detailed balance theory of fluorescent collectors is compatible with the SQ approach ${ }^{1}$ to conventional solar cells.

\section{OPTOELECTRONIC RECIPROCITY}

We now proceed to calculate the photon flux density $\phi_{e m}$ emitted from the cell surface if the device is operated as an LED, i.e., excess carriers are injected via the junction into the device. The light production at a coordinate $\mathbf{r}$ in the volume of the device is given by the product of excess minority carriers and the radiative recombination rate. Thus, we have instead of Eq. (17),

$$
\frac{d \phi}{d \mathbf{r}}(\mathbf{r})=\alpha(\mathbf{r}) n^{2}(\mathbf{r}) \phi_{b b} u(\mathbf{r}) .
$$

Let us now assume that the coordinate $\mathbf{r}$ is found on the optical path from a surface element at coordinate $\mathbf{r}_{S}$ into a spatial angle $d \Omega\left(\mathbf{r}_{S}, \mathbf{r}\right)$ defined as the angle at which the volume element $d A(\mathbf{r}) d \mathbf{r}$ is seen from the outside of the cell [cf. Fig. 2(b)]. By analogy with Eq. (12), we have

$$
\phi_{e m}\left(\mathbf{r}_{S}\right) d \Omega\left(\mathbf{r}_{S}, \mathbf{r}\right)=T\left(\mathbf{r}, \mathbf{r}_{S}\right) \phi(\mathbf{r}) d \Omega\left(\mathbf{r}, \mathbf{r}_{S}\right)
$$

From Eq. (22), the volume element located at $\mathbf{r}$ contributes to the emission at $\mathbf{r}_{S}$ by an amount

$$
\begin{aligned}
\phi_{e m}\left(\mathbf{r}_{S}\right) & =T\left(\mathbf{r}, \mathbf{r}_{S}\right) \alpha(\mathbf{r}) n^{2}(\mathbf{r}) \frac{d \Omega\left(\mathbf{r}, \mathbf{r}_{S}\right)}{d \Omega\left(\mathbf{r}_{S}, \mathbf{r}\right)} \phi_{b b} u(\mathbf{r}) d \mathbf{r} \\
& =T\left(\mathbf{r}, \mathbf{r}_{S}\right) \alpha(\mathbf{r}) n^{2}(\mathbf{r}) \frac{n^{2}\left(\mathbf{r}_{S}\right)}{n^{2}(\mathbf{r})} \phi_{b b} u(\mathbf{r}) d \mathbf{r} .
\end{aligned}
$$

Integrating Eq. (22) over the optical path, with $n\left(\mathbf{r}_{S}\right)$ being the refractive index of the ambient, i.e., $n\left(\mathbf{r}_{S}\right)=1$, we have

$$
\phi_{e m}\left(\mathbf{r}_{S}, \Omega_{S}, E_{\gamma}\right)=\int T\left(\mathbf{r}, \mathbf{r}_{S}\right) \alpha(\mathbf{r}) \phi_{b b}\left(E_{\gamma}\right) u(\mathbf{r}) d \mathbf{r} .
$$

By means of Donolato's reciprocity theorem [Eq. (8)] and the symmetry $T\left(\mathbf{r}, \mathbf{r}_{S}\right)=T\left(\mathbf{r}_{S}, \mathbf{r}\right)$, we finally obtain 


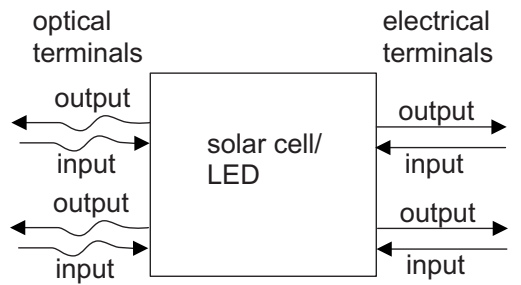

FIG. 3. A solar cell or LED viewed as a multiprobe device. The inputs and outputs of the electrical terminals and the optical terminals are linked by reciprocity relations.

$$
\begin{aligned}
\phi_{e m}\left(\mathbf{r}_{S}, \Omega_{S}, E_{\gamma}\right)= & \int T\left(\mathbf{r}_{S}, \mathbf{r}\right) \alpha(\mathbf{r}) f_{c}(\mathbf{r}) d \mathbf{r} \phi_{b b}\left(E_{\gamma}\right) \\
& \times\left[\exp \left(\frac{q V}{k T}\right)-1\right] .
\end{aligned}
$$

In the next step, we follow an incoming photon flux $\phi_{\text {in }}\left(\mathbf{r}_{S}\right) \delta \Omega_{\text {in }}$ that propagates along the same optical path in the inverse direction, such that $\Phi(\mathbf{r})=T\left(\mathbf{r}_{S}, \mathbf{r}\right) \phi_{\text {in }}\left(\mathbf{r}_{S}\right) \delta \Omega_{\text {in }}$ at each point. This photon flux will generate a short circuit current,

$$
\begin{aligned}
\delta J_{S C} & =q \int \alpha(\mathbf{r}) \Phi(\mathbf{r}) f_{C}(\mathbf{r}) d \mathbf{r} \\
& =\phi_{\text {in }}\left(\mathbf{r}_{S}\right) \delta \Omega_{\text {in }} \int T\left(\mathbf{r}_{S}, \mathbf{r}\right) \alpha(\mathbf{r}) f_{C}(\mathbf{r}) d \mathbf{r} .
\end{aligned}
$$

From the definition of the (partial) quantum efficiency, we obtain

$$
Q\left(\mathbf{r}_{S}, \theta, \varphi, E_{\gamma}\right):=\frac{\delta J_{S C} / q}{\phi_{i n}\left(\mathbf{r}_{S}\right) \delta \Omega_{i n}}=\int T\left(\mathbf{r}_{S}, \mathbf{r}\right) \alpha(\mathbf{r}) f_{c}(\mathbf{r}) d \mathbf{r} .
$$

Inserting Eq. (28) into Eq. (26) yields Eq. (6), i.e., the reciprocity relation between the photovoltaic quantum efficiency and electroluminescent emission of solar cells and LEDs.

In practice, the external photovoltaic quantum efficiency $\mathrm{EQE}_{P V}\left(E_{\gamma}\right)$ is measured at normal incidence. For this situation, we may reformulate Eq. (6) as

$$
\phi_{e m}^{\text {norm }}\left(E_{\gamma}\right)=\operatorname{EQE}_{P V}\left(E_{\gamma}\right) \phi_{b b}\left(E_{\gamma}\right)\left[\exp \left(\frac{q V}{k T}\right)-1\right],
$$

which relates the luminescence emission $\phi_{e m}^{n o r m}\left(E_{\gamma}\right)$ normal to the surface of the device to the $\mathrm{EQE}_{P V}$.

Equation (29) thus connects the optical output of a solar cell and its electrical input and vice versa. This suggests a picture of a solar cell or LED, as shown in Fig. 3, that is inspired by the electrical multichannel systems analyzed by Büttiker et al. ${ }^{24}$ In a solar cell or LED, we have not only one or more electrical terminals or probes but also optical terminals according to light of different wavelengths. The interchanging of electrical input/output and optical input/output is regulated by the above reciprocity relation. The reciprocity between different optical terminals in Fig. 3 is described by Würfel's generalization of Kirchhoff's law, ${ }^{25}$ which will be discussed in Sec. VII. The electrical terminals are interre- lated by Büttiker's reciprocity. ${ }^{26,27}$ Note that an early version of the reciprocity between the emitter and collector currents of bipolar transistors was described by Shockley et al. ${ }^{28}$ in 1951.

\section{OPEN CIRCUIT VOLTAGE AND LIGHT EMITTING DIODE QUANTUM EFFICIENCY}

It is worthwhile pointing out that the integral reciprocity relation [Eq. (7)] yields a detailed balance theory that not only extends previous reciprocity relations but also generalizes the SQ approach to less idealized situations allowing for finite nonradiative lifetimes $\tau_{n r}$ and finite charge carrier mobilities. This is because knowledge of $Q\left(\mathbf{r}_{S}, \theta, \varphi, E_{\gamma}\right)$ enables both the calculation of the short circuit current $J_{S C}$ by integration of Eq. (2) and $J_{0, e m}$ from Eq. (7). In turn, $J_{S C}$ and $J_{0, e m}$ enable us to derive the radiative limit,

$$
V_{O C}^{r a d}=\frac{k T}{q} \ln \frac{J_{S C}}{J_{e m, 0}},
$$

for the open circuit voltage of a specific device directly from experimental data.

However, the measured open circuit voltage $V_{O C}$ will equal $V_{O C}^{r a d}$ only if the nonradiative (or more precisely nonemitting) recombination current $J_{n e}(V)$ at a given voltage $V$ is zero. This is precisely the case if the external quantum efficiency,

$$
\operatorname{EQE}_{L E D}(V)=\frac{J_{e m}(V)}{J_{e m}(V)+J_{n e}(V)}=\frac{J_{e m}(V)}{J_{i n j}(V)},
$$

in the LED sense is unity. Here, $J_{i n j}(V)=J_{n e}(V)+J_{e m}(V)$ denotes the current injected in the dark by the applied voltage bias $V$. In the presence of nonradiative recombination, we have $J_{n e}(V)>0$ and hence $\mathrm{EQE}_{L E D}<1$.

The latter situation can be expressed quantitatively by using the diode law,

$$
J_{e m}=J_{e m, 0}\left[\exp \left(\frac{q V}{k T}\right)-1\right] \approx J_{e m, 0} \exp \left(\frac{q V}{k T}\right),
$$

for the radiative emission current. Combining the logarithm of Eq. (31) with Eq. (32) at a bias voltage $V=V_{O C}$ yields

$$
\begin{aligned}
\ln \left(\mathrm{EQE}_{L E D}\right) & =\ln \left[\frac{J_{e m, 0} \exp \left(q V_{O C} / k T\right)}{J_{i n j}\left(V_{O C}\right)}\right] \\
& =\frac{q V_{O C}}{k T}+\ln \left[\frac{J_{e m, o}}{J_{i n j}\left(V_{O C}\right)}\right] .
\end{aligned}
$$

In the open circuit situation, we have the equality between the injected current $J_{i n j}\left(V_{O C}\right)$ and the short circuit current $J_{S C}$ (cf. Fig. 4) such that the total current $J_{t o t}(V)=J_{i n j}(V)-J_{S C}$ amounts to zero. With $J_{i n j}\left(V_{O C}\right)=J_{S C}$ and Eq. (30), we finally obtain

$$
\Delta V_{O C}=V_{O C}^{r a d}-V_{O C}=-\frac{k T}{q} \ln \left(\mathrm{EQE}_{L E D}\right) .
$$

Figure 4 illustrates Eq. (34) by showing that the radiative current $J_{e m}(V)$ has a slope of unity in a semilogarithmic plot 


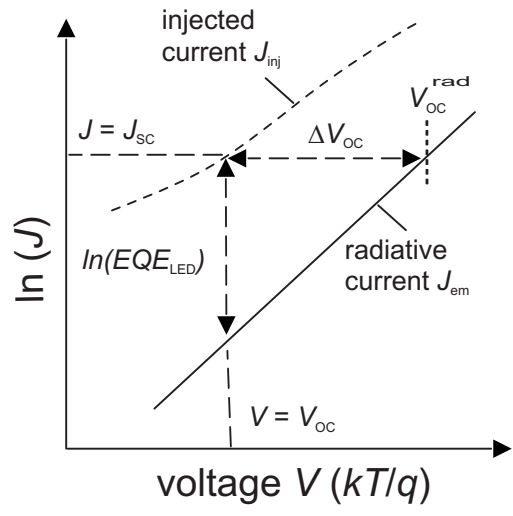

FIG. 4. Semilogarithmic plot of the total current $J_{t o t}(V)$ (dotted line) and the radiative current $J_{e m}(V)$ (full line) as a function of voltage $V$ normalized to $k T / q$. The radiative current $J_{e m}(V)$ has a slope of unity in this representation. Therefore, the vertical distance (at constant $V$ ) and the horizontal distance (at constant $J$ ) between any point of the $J_{t o t}(V)$ and $J_{e m}(V)$ characteristics are equal. Thus, we have at any bias point $-\ln \left(\mathrm{EQE}_{L E D}\right)=\ln \left[J_{t o t}(V) / J_{e m}(V)\right]$ $=q \Delta V_{O C} / k T$.

if the voltage is normalized to the thermal voltage $k T / q$. Therefore, on this scaling, the distance between any point of the real current voltage characteristics $J_{i n j}(V)=J_{t o t}(V)+J_{S C}$ in the vertical direction, given by $\ln \left(J_{i n j} / J_{e m}\right)=-\ln \left(\mathrm{EQE}_{L E D}\right)$, equals the distance in the horizontal direction, i.e., $\left(V_{O C}^{r a d}\right.$ $\left.-V_{O C}\right) /(k T / q)$.

Equation (34) is a reciprocity relation in its own right, as it connects the (LED) external quantum efficiency $\mathrm{EQE}_{L E D}$ with the open circuit voltage $V_{O C}$ of a solar cell. Equation (34) is complementary to Eq. (6) as it allows the open circuit voltage $V_{O C}$ to be derived from the luminescent properties of the same device. Analogously, we can in principle determine the short circuit current $J_{S C}$ from the electroluminescent emission $\phi_{e m}\left(E_{\gamma}\right)$ with the help of Eq. (6). Thus, we can derive all essential photovoltaic parameters from knowledge of the electroluminescent properties of the device. Equivalently, we can calculate the EL spectrum and the luminescence efficiency from purely photovoltaic analysis, i.e., the knowledge of the quantum efficiency $\mathrm{EQE}_{P V}$ and of the open circuit voltage $V_{O C}$.

\section{VALIDITY OF THE RECIPROCITY RELATIONS}

In the following, we denote the reciprocity between electroluminescent emission and photovoltaic quantum efficiency as theorem I, given in different versions by Eq. (6) and (7), or (29). The reciprocity between open circuit voltage and the electroluminescent quantum efficiency given by Eq. (34) will be denoted as theorem II.

As pointed out above, the validity of theorem I follows from the reciprocity of photonic interaction between the ambient and the photovoltaic absorber as well as from the reciprocity of photonic self-interaction within the absorber both combined with Donolato's reciprocity for carrier transport. ${ }^{8}$ Thus, theorem I is a consequence of the principle of detailed balance. However, this principle is strictly valid only in ther- mal equilibrium and may be extended to nonequilibrium situations only if the departure from equilibrium is linear. In classical, bipolar solar cells, nonlinear recombination terms containing the product of the electron and the hole concentration may dominate the device behavior (e.g., in pinjunction devices). This situation leads to the breakdown of Donolato's theorem in its integral form as it has been shown in Ref. 15. In organic solar cells, the concentration of excitons plays a dominant role, ${ }^{14}$ though electrons and holes must be present after charge separation. However, if the concentration and the transport of excitons play a dominant role, the charge separating interface(s) as defined in Ref. 14 plays the identical role as the separating junction in above derivation. Therefore, reciprocity theorems I and II should be valid as long as nonlinear effects, e.g., given by the interaction of excitons with electrons and holes, can be neglected. Ongoing research $^{29}$ suggests that in excitonic solar cells, this is a reasonable approach at least in some relevant cases.

We note further that the validity of theorem I is a sufficient condition for the so-called "superposition principle" that states that the illuminated current-voltage characteristics $J(V)$ of a solar cell are composed by the voltage dependence of the dark injection current $J_{D}(V)$ and the short circuit current $J_{S C}$ according to

$$
J(V)=J_{D}(V)-J_{S C} .
$$

In turn, the superposition principle enters into the proof of reciprocity theorem II as given in the previous section.

\section{ROLE OF MOBILITY AND LIFETIMES IN LIGHT EMITTING DIODES AND SOLAR CELLS}

A first application of reciprocity relations I and II is prompted by our initial question on the difference between low-mobility and long-lifetime materials and high-mobility and low-lifetime materials. Let us firstly assume a lowmobility $\mu_{p}$ of the relevant species (electrons, holes, or excitons) but no nonradiative recombination, i.e., $\tau_{n r}=\infty$. In the worst case, the mobility might be so low that only a small fraction of the photogenerated charge carriers is collected by the junction. Likewise, in organic solar cells, the mobility of excitons might be so low that only a small fraction arrives at the interfaces that cause their dissociation into electrons and holes. In any case, such a low-mobility device has a poor photovoltaic quantum efficiency $\mathrm{EQE}_{P V}$ and, consequently, a poor short circuit current $J_{S C}$ and output power $P$. The output power of a solar cell is given by

$$
P=J_{S C} V_{O C} F F,
$$

where the fill factor $F F$, to first order, only depends on the open circuit voltage $V_{O C} \cdot{ }^{30}$

Because of the assumed absence of nonradiative recombination, the noncollected charge carriers (or excitons) must recombine radiatively and reemit photons. Thus, in contrast to the ideal SQ case where short circuiting the solar cell completely quenches photoluminescence (PL), the lowmobility device will be photoluminescent under both open circuit and short circuit conditions. Such a situation is encountered in organic solar cells unless an admixture of com- 
ponents, the essential ingredient to boost the efficiency of organic solar cells, ${ }^{31}$ leads to PL quenching that is now, however, independent of the bias conditions. A low-mobility material alone is thus unlikely to yield high-efficiency solar cells because a low short circuit current enters linearly in the output power $P$ of the solar cell [Eq. (35)].

In contrast, as long as the nonradiative (nonemitting) recombination current $J_{n e}(V)$ at a given bias voltage $V$ is smaller than the emitted current $J_{e m}(V)$, the present theorem II predicts a reasonably high external LED quantum efficiency $\mathrm{EQE}_{L E D}$. Therefore, low-mobility materials with a low portion of nonemitting recombination may yield highly efficient LEDs.

Turning now to the other departure from the SQ situation, we consider a semiconductor with a low nonradiative lifetime $\tau_{n r}$ but a mobility that is high enough to enable perfect carrier collection. Such a device would have an $\mathrm{EQE}_{P V}$ that is close to unity in the relevant spectral range. Operating as an LED, such a device would emit more photons at a given bias voltage than a device built from a low-mobility material with low $\mathrm{EQE}_{P V}$ (cf. theorem I). However, as $J_{n e}$ is much larger than $J_{e m}$, we obtain a poor $\mathrm{EQE}_{L E D}$, making the material rather unsuitable as an LED. However, this low-lifetime material may still yield a very good solar cell because the high mobility results in a high $\mathrm{EQE}_{P V}$ and hence a high $J_{S C}$. The measured open circuit voltage $V_{O C}$ of this device will, however, be smaller than the radiative limit as predicted by theorem II. This loss might be acceptable because $\mathrm{EQE}_{L E D}$ enters only logarithmically into Eq. (33) and consequently also in Eq. (34). Therefore, even a relatively low $\mathrm{EQE}_{L E D}$ still yields a reasonably high $V_{O C}$, as demonstrated specifically by high-efficiency silicon solar cells. ${ }^{6}$ A more quantitative exploration of these points is given in Ref. 32 and direct experimental evidence for the validity of Eqs. (29) and (34) from highly efficient $\mathrm{Si}$ and $\mathrm{Cu}(\mathrm{In}, \mathrm{Ga}) \mathrm{Se}_{2}$ solar cells is shown in Ref. 33.

\section{RELATIONS BETWEEN PHOTOLUMINESCENCE AND ELECTROLUMINESCENCE}

There exist close analogies between the present electrooptical reciprocity relations and the analysis of optoelectronic materials by PL measurements. In the latter case, the reciprocity between the potential open circuit voltage, i.e., the actual splitting $\mu$ of the quasi-Fermi levels, and the PL emission is given by Würfel's generalization of Kirchhoff's law. ${ }^{25}$ For the case of emission normal to the semiconductor surface, this may be written as

$$
\phi_{P L}\left(E_{\gamma}\right) \approx a\left(E_{\gamma}\right) \phi_{b b}\left(E_{\gamma}\right)\left[\exp \left(\frac{\mu}{k T}\right)-1\right],
$$

where $\phi_{P L}\left(E_{\gamma}\right)$ is the excess PL intensity, $a\left(E_{\gamma}\right)$ the absorptance of the semiconductor at a given photon energy $E_{\gamma}$, and $\mu$ is the split of quasi-Fermi levels that equals the chemical potential of photons. ${ }^{25}$ Application of Eq. (37) to a combination of PL and absorption measurements yields valuable information on the maximum open circuit voltage that may be realized in $\mathrm{Si}$ wafers ${ }^{34}$ and $\mathrm{Cu}(\mathrm{In}, \mathrm{Ga}) \mathrm{Se}_{2}$ thin films. ${ }^{35}$

It is interesting to note that replacing $\mu$ by $q V$ and $a\left(E_{\gamma}\right)$ by $\mathrm{EQE}_{P V}\left(E_{\gamma}\right)$ in Eq. (37) leads to Eq. (29). The analogy between the present reciprocity given by Eq. (29) and Würfel's equation demonstrates the similarities and differences between the reciprocity in PL and EL. The role of the splitting of the quasi-Fermi levels in PL is played by the junction voltage $V$ for the case of EL. Note that in the latter case, the quantity $\mu$ equals $q V$ only at the location of the junction and that $\mu$ may thus vary freely according to the restrictions of electronic transport without violating the reciprocity relation. For PL, the prefactor $a\left(E_{\gamma}\right)$ in Eq. (37) only reflects the optical properties of the semiconductor, $\mathrm{EQE}_{P V}\left(E_{\gamma}\right)$, whereas the analogous quantity in Eq. (29) contains the absorptance and in addition the probability of charge carrier collection within the device. The inequality $\mathrm{EQE}_{P V}\left(E_{\gamma}\right) \leqslant a\left(E_{\gamma}\right)$ results from the fact that $\mathrm{EQE}_{P V}\left(E_{\gamma}\right)$ considers the series connection of light absorption and carrier collection. In a similar way, reciprocity theorem I [Eqs. (6), (7), and (29)] results from the series connection of the detailed balance pair light absorption and emission with the pair carrier collection and injection.

\section{CONCLUSIONS}

The present paper has developed two basic reciprocity relations between the photovoltaic and the electroluminescent actions of solar cells and LEDs. The first relates the spectral and angular dependence of the electroluminescence of solar cells and light emitting diodes to the spectral and angular photovoltaic external quantum efficiency. The second reciprocity relation connects the open circuit voltage of a solar cell and its EL quantum efficiency. Combination of both relations allows for a detailed characterization of the photovoltaic properties of a device by measuring its electroluminescent properties and vice versa.

In view of the considerable volume of work analyzing photovoltaic devices by means of EL techniques, ${ }^{36-38}$ it is important to note that the validity of both reciprocity relations requires no special assumptions on the nature of the nonradiative recombination mechanism dominating the current voltage. We only need the validity of the principle of detailed balance for the radiative part of the recombination current. Thus, both reciprocity relations should have a wide range of applicability.

\section{ACKNOWLEDGMENTS}

The author is grateful to T. Kirchartz, J. Mattheis, S. Reynolds, and J. H. Werner for valuable discussions and arguments. This work was supported by a grant from the German Ministry for Research and Technology (Contract No. 03SF0308E). 
*Corresponding author. u.rau@fz-juelich.de

${ }^{1}$ W. Shockley and H. J. Queisser, J. Appl. Phys. 32, 510 (1961).

${ }^{2}$ M. A. Green, J. Zhao, A. Wang, P. J. Reece, and M. Gal, Nature (London) 412, 805 (2001).

${ }^{3}$ For a recent review, see R. H. Friend, R. W. Gymer, A. B. Holmes, J. H. Burroughes, R. N. Marks, C. Taliani, D. D. C. Bradley, D. A. Dos Santos, J. L. Bredas, M. Logdlund, and W. R. Salaneck, Nature (London) 397, 121 (1999).

${ }^{4}$ C. Adachi, M. A. Baldo, S. R. Forrest, and M. E. Thompson, Appl. Phys. Lett. 77, 904 (2000).

${ }^{5}$ For a recent review, see J. Nelson, in Practical Handbook of Photovoltaics, edited by T. Markvart and L. Castaner (Elsevier, Oxford, 2003) p. 848; Organic Photovoltaics: Concepts and Realization, edited by C. Brabec, V. Dyakonov, J. Parisi, and N. S. Sariciftci (Springer, Berlin, 2003).

${ }^{6}$ J. H. Zhao, A. H. Wang, M. A. Green, and F. Ferrazza, Appl. Phys. Lett. 73, 1991 (1998).

${ }^{7}$ W. Shockley, Bell Syst. Tech. J. 28, 435 (1949).

${ }^{8}$ C. Donolato, Appl. Phys. Lett. 46, 270 (1985).

${ }^{9}$ K. Misiakos and F. A. Lindholm, J. Appl. Phys. 58, 4743 (1985).

${ }^{10}$ C. Donolato, J. Appl. Phys. 66, 4524 (1989).

${ }^{11}$ T. Markvart, IEEE Trans. Electron Devices ED-43, 1034 (1996).

${ }^{12}$ M. A. Green, J. Appl. Phys. 81, 268 (1997).

${ }^{13}$ J. A. del Alamo and R. M. Swanson, IEEE Trans. Electron Devices ED-31, 1878 (1984).

${ }^{14}$ B. A. Gregg, J. Phys. Chem. B 107, 4688 (2003).

${ }^{15}$ U. Rau and R. Brendel, J. Appl. Phys. 84, 6412 (1998).

${ }^{16}$ W. P. Dumke, Phys. Rev. 105, 139 (1957).

${ }^{17}$ A. Martì, J. L. Balenzategui, and R. F. Reyna, J. Appl. Phys. 82, 4067 (1997).

${ }^{18}$ W. Van Roosbroeck and W. Shockley, Phys. Rev. 94, 1558 (1956).

${ }^{19}$ T. Kuriyama, T. Kamiya, and H. Yanai, Jpn. J. Appl. Phys. 16, 465 (1977).
${ }^{20}$ K. Mettler, Phys. Status Solidi A 49, 163 (1978).

${ }^{21}$ A. Goetzberger and V. Wittwer, Sol. Cells 4, 3 (1981).

${ }^{22}$ U. Rau, F. Einsele, and G. C. Glaeser, Appl. Phys. Lett. 87, 171101 (2005).

${ }^{23}$ T. Markvart, J. Appl. Phys. 99, 026101 (2006).

${ }^{24}$ M. Büttiker, Y. Imry, R. Landauer, and S. Pinhas, Phys. Rev. B 31, 6207 (1985).

${ }^{25}$ P. Würfel, J. Phys. C 15, 3967 (1982).

${ }^{26}$ M. Büttiker, Phys. Rev. Lett. 57, 1761 (1986).

${ }^{27}$ M. Büttiker, IBM J. Res. Dev. 32, 317 (1988).

${ }^{28}$ W. Shockley, M. Sparks, and G. K. Teal, Phys. Rev. 83, 151 (1951).

${ }^{29}$ T. Kirchartz and U. Rau (unpublished).

${ }^{30}$ M. A. Green, Solar Cells: Operating Principles, Technology and System Applications (Prentice-Hall, Englewood Cliffs, NJ, 1982), p. 96.

${ }^{31}$ N. S. Sariciftci, L. Smilowitz, A. J. Heeger, and F. Wudl, Science 258, 1474 (1992).

${ }^{32}$ J. Mattheis, U. Rau, and J. H. Werner, Conference Record of the 2006 IEEE Fourth World Conference on Photovoltaic Energy Conversion (IEEE, New York, 2006), p. 95.

${ }^{33}$ T. Kirchartz, U. Rau, M. Kurth, J. Mattheis, and J. H. Werner, Thin Solid Films 515, 6238 (2007).

${ }^{34}$ S. Finkbeiner, E. Daub, and P. Würfel, 11th European Photovoltaic Solar Energy Conference (Harwood Academic, Montreux, 1992), pp. 320-322.

${ }^{35}$ G. H. Bauer, R. Brüggemann, S. Tardon, S. Vignoli, and R. Kniese, Thin Solid Films 480-481, 410 (2005).

${ }^{36}$ T. Trupke, E. Daub, and P. Würfel, Sol. Energy Mater. Sol. Cells 53, 103 (1998).

${ }^{37}$ T. Trupke, P. Würfel, I. Uhlendorf, and I. Lauermann, J. Phys. Chem. B 103, 1905 (1999).

${ }^{38}$ T. Fuyuki, H. Kondo, T. Yamazaki, Y. Takahashi, and Y. Uraoka, Appl. Phys. Lett. 86, 262108 (2005). 